\title{
Catalysis Process Development for Hydrogen Storage/Generation with LOHCs
}

\author{
Ji Su ${ }^{1} \cdot$ Jiaxu Liu ${ }^{2}$ \\ Accepted: 18 June 2021 / Published online: 2 July 2021 \\ (c) The Author(s), under exclusive licence to Springer Science+Business Media, LLC, part of Springer Nature 2021
}

The hydrogen as green energy carrier is an envisioned future. Hydrogen storage is a key enabling technology for the advancement of hydrogen and fuel cell technologies in applications including stationary power, portable power, and transportation. Hydrogen has the highest energy per mass of any fuel; however, its low ambient temperature density results in a low energy per unit volume, therefore requiring the development of advanced storage methods that have potential for higher energy density. Using chemical bonds of liquid organic hydrogen carriers (LOHCs) to storage and re-generate hydrogen are of great merits, including not only high reversibility, selectivity, gravimetric and volumetric hydrogen density, but also low investment risk owing to the high compatibility with current transport infrastructure.

Research efforts on LOHCs seek practical and novel carrier systems and advanced catalytic materials that have the potential to reduce costs, increase reaction rate, and provide a more efficient catalytic hydrogen generation/storage process. Within this special issue, the most recent research advances in the LOHCs process and catalysts development have been included herein and systematically reviewed. The Guest Editors Dr. Ji Su and Prof. Jiaxu Liu sincerely thank the authors for submitting their high-quality work to this special issue, especially during this COVID-19 pandemic which indeed influenced and slowed down everything. The great help and detailed comments from many reviewers are highly appreciated. We are also very thankful to the generous support from Editor Gabor A. Somorjai and Springer Nature editorial team during the whole publication process.

\footnotetext{
Ji Su

Jisu@lbl.gov

$\triangle$ Jiaxu Liu

liujiaxu@dlut.edu.cn

1 The Molecular Foundry, Lawrence Berkeley National Laboratory, Berkeley, CA 94720, USA

2 Industrial Catalysis, State Key Laboratory of Fine Chemicals, Dalian University of Technology, Dalian 116023, Liaoning, China
}

To develop practical LOHCs process, we urge the researchers in the field to pay more attention on the stability of both the catalyst and LOHC molecules. Chiyoda has successfully developed the proprietary dehydrogenation catalyst for methylcyclohexane (MCH) and has conducted technological demonstrations to make the catalyst for practical use. Chiyada's $\mathrm{Pt} / \mathrm{Al}_{2} \mathrm{O}_{3}$ catalyst provide a threshold of catalyst stability (above $10 \mathrm{k}$ hours) for the researchers to refer. Furthermore, the durability of the catalyst after regeneration and recycling is important. On the other hand, the stability of the LOHC molecules affect the efficiency of the $\mathrm{H}_{2}$ storage and evolution, reaction selectivity, especially, the undesired decomposition by products increases the difficulty of subsequent $\mathrm{H}_{2}$ separation and purification.

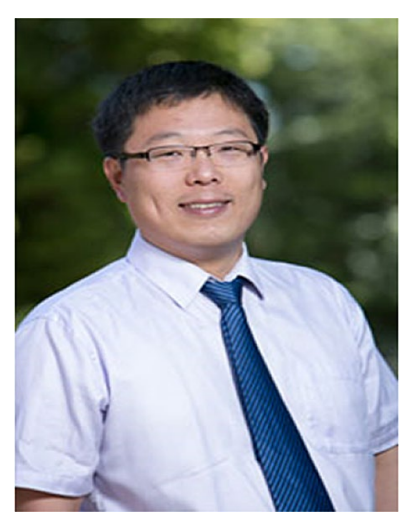

Dr. Ji Su

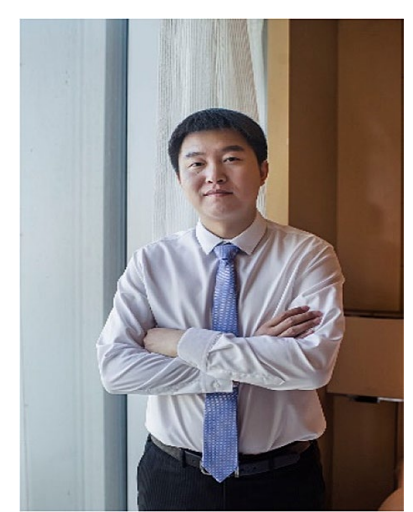

Dr. Jiaxu Liu
Publisher's Note Springer Nature remains neutral with regard to jurisdictional claims in published maps and institutional affiliations. 